\title{
A Local Extension of the Electrophilicity Index Concept
}

\author{
Andrés Cedillo*1 and Renato Contreras ${ }^{2}$
}

1 Departamento de Química, Universidad Autónoma Metropolitana-Iztapalapa, San Rafael Atlixco 186, Iztapalapa D.F. 09340, México.cedillo@xanum.uam.mx

2 Departamento de Química, Facultad de Ciencias, Universidad de Chile, Casilla 653-Santiago, CHILE. rcontrer@argon. ciencias.uchile.cl

Received December 05, 2011; accepted March 06, 2012

\begin{abstract}
A local measure of the electrophilicity has been recently proposed to analyze the chemical reactivity of several kinds of molecules. In this work a theoretical rationalization of the local extension is proposed following the quantitative definition of the molecular electrophilic power and a variational method for the distribution of the transferred charge. A condensation scheme to atoms or fragments follows from its relation to the Fukui function and the local softness. Differences between these quantities are discussed and they are tested in a model system. The analysis shows that the local electrophilicity is more appropriate to describe differences among a set of substituted molecules.
\end{abstract}

Key words: Chemical Reactivity, Local Descriptors, Electrophilicity, Density Functional Theory.

\section{Introduction}

Interactions between electrophiles and nucleophiles are present in many chemical systems. In a charge transfer process, the electrophile receives charge from the nucleophile, therefore, in many of these cases, the electrophile acts as a Lewis acid whereas the nucleophile behaves as a Lewis base. This kind of interactions gave rise to chemical concepts like electronegativity and frontier orbital theory.

The prediction of the reactivity of chemical species is one of the main purposes of theoretical chemistry and a lot of work has been done on this line. In particular, density functional theory (DFT) [1] has provided formal definitions for many chemical concepts [2] like electronegativity $(\chi)$ [3], chemical hardness $(\eta)$ [4], Fukui function $(f(\mathbf{r}))$ [5], global $(S)$ and local softness $(s(\mathbf{r}))$ [6], and, in consequence, quantitative scales of these properties. The study of the reactivity within the DFT framework has also given more complex quantities, like the hardness and softness kernels [7], but the reactivity picture is far from being complete.

From an empirical relationship [8], an electrophilicity index, $\omega$, was recently developed using DFT [9]. The quantification of the electrophilicity concept is based on the maximum energetic stabilization of a species that arises from accepting charge. The energy, $E$, of an electronic system depends on the number of electrons, $N$, and the external potential, $v(\mathbf{r})$. In an isolated molecule, the external potential corresponds to the electric potential of the nuclei. When the external potential remain fixed, the energy is only modified if the number of electrons changes. If we use a Taylor series expansion up to second order, the energy change can be written in the following form,
Resumen. Recientemente se propuso una medida de la electrofilia a nivel local para analizar la reactividad química de diferentes familias de moléculas. En este trabajo se presenta un modelo teórico que justifica dicha extensión al ámbito local, partiendo de la definición del índice de electrofilia y del método variacional para la distribución de carga transferida. A partir de la relación con la función de Fukui y la blandura local, también se tiene un esquema de condensación por átomos o fragmentos. Se describen las diferencias entre estos índices locales y se prueban en un sistema modelo. El análisis muestra que la electrofilia local es un mejor índice para describir las diferencias dentro de un conjunto de moléculas sustituidas.

Palabras clave: Reactividad química, índices locales, electrofilia, teoría de funcionales de la densidad.

$$
\begin{aligned}
\Delta E & \equiv E[N+\Delta N, v]-E[N, v] \\
& \approx\left(\frac{\partial E}{\partial N}\right)_{v} \Delta N+\frac{1}{2}\left(\frac{\partial^{2} E}{\partial N^{2}}\right)_{v}(\Delta N)^{2} \\
& \approx \mu \Delta N+\frac{1}{2} \eta(\Delta N)^{2}
\end{aligned}
$$

where the chemical potential, $\mu$, is the derivative of the energy with respect to the number of electrons and corresponds to the negative of the electronegativity [3], and the chemical hardness, $\eta$, is the corresponding second derivative [6]. Since an electrophile is a chemical species capable of accepting electrons, its energy must decrease when it receives an electron, then its chemical potential must be negative, while the hardness is positive. As the electrophile accepts charge, its accepting capability will decrease up to a state that is unable to receive more electrons. Let $\Delta N_{\max }$ and $\omega \equiv-\Delta E_{\min }$ stand for that number of electrons and the corresponding energetic stabilization, respectively. From the quadratic approximation given by eq. 1, $\Delta E_{\min }$ corresponds to the minimum value of the quadratic function, then the energetic stabilization becomes

$$
\omega=\frac{1}{2} \mu^{2} S=-\frac{1}{2} \mu \Delta N_{\max }
$$

and the amount of transferred charge is given by

$$
\Delta N_{\max }=-\mu S,
$$

where we used the inverse relation between the hardness and the softness, $S=1 / \eta$ [6]. Parr, von Szentpaly and Lui [9] associated the electrophilic power to the value of $\omega$. Note that the electrophilicity index, $\omega$, is a positive global quantity which is linearly related to $\Delta N_{\max }$ and the electronegativity, therefore 
both quantities, which constitute a characteristic feature of the electrophiles, contribute in the same amount to the value of $\omega$. In addition, the electrophilicity index encompasses, apart of the propensity of the electrophile to acquire an additional electronic charge driven by the chemical potential, $\mu$, the resistance of the system to exchange electronic charge with its environment described by the chemical hardness, $\eta=1 / S$. A good electrophile is therefore characterized by a high value of $\mu$ and a low value of $\eta$.

Recently a local version of this electrophilic power has been proposed to analyze the reactivity of several molecules [10]. This local index distributes the electrophilicity index $\omega$ on the molecule using the Fukui function, $w(\mathbf{r})=\omega f(\mathbf{r})$, and it was empirically proposed as an analogy of the relation between the molecular softness, $S$, and the local softness, $s(\mathbf{r})=S f(\mathbf{r})$.

In this work we present a theoretical rationalization to the use of this local extension based on the application of a variational principle.

\section{Model}

An electrophile may have many electron-attracting sites, but when it receives additional charge, the sites do not equally contribute to the charge stabilization. For a given amount of charge transfer $(\Delta N)$, it was recently shown [11] that the most stable way to distribute the additional charge corresponds to $\Delta \rho(\mathbf{r})=\Delta N f(\mathbf{r})$. That is, an additional charge must be distributed according to the Fukui function in order to minimize the molecular energy. This variational principle for the way of distributing additional charge in a molecule, or the way of extracting an amount of charge from a molecule, directly follows from the energy variational principle [11].

When an electrophile receives the maximum amount of electron transfer $\left(\Delta N_{\max }\right)$, this additional charge must be distributed following the Fukui function, and consequently, in any region of space, the change of the density will be equal to

$$
\Delta \rho(\mathbf{r})=\Delta N_{\max } f(\mathbf{r}) .
$$

Since the chemical potential is the same all along the molecule, every point in space contributes to the electrophilicity index in the following amount,

$$
\begin{aligned}
\omega(\mathbf{r}) & \equiv-\frac{1}{2} \mu \Delta \rho(\mathbf{r})=\frac{1}{2} \mu^{2} S f(\mathbf{r}) \\
& =\omega f(\mathbf{r})=\frac{1}{2} \mu^{2} s(\mathbf{r})
\end{aligned}
$$

where $f(\mathbf{r})$ and $s(\mathbf{r})$ correspond to the Fukui function and the local softness, respectively. If one integrates $w(\mathbf{r})$ over all the space the electrophilicity index is obtained,

$$
\omega=\int w(\mathbf{r}) d \mathbf{r} .
$$

Therefore one can designate $w(\mathbf{r})$ as the local electrophilicity index, associated with the global quantity $\omega$. It is important to mention that an electrophile is an electron acceptor species, and then both the Fukui function and the local softness, in eq. 5, must be calculated in the direction of an increase in the number of electrons.

From eq. 5, one can note that the local electrophilicity, the Fukui function and the local softness are linearly related, and the three local quantities give the same relative information when two different regions of the same molecule are analyzed. This similarity comes from the fact that all those quantities are related with the response of the density under changes in the number of particles. Beyond the similarity, the difference in the scale factor makes them useful for different kind of analysis. For a single molecule, the more reactive sites correspond to those regions with high values of any of the three quantities. The relative value between the reactive sites is the same since all the quantities are proportional. If one compares a set of different molecules, Fukui function is not a good choice. Fukui function integrates to unity and, in many cases, it distributes over all the sites of the molecule giving very different values for molecules of different sizes but with similar chemical behavior. Therefore both the local softness and the local electrophilicity are more suitable for the comparison in a set of molecules. There are some cases, like a series of substituted molecules, where the softnesses are very similar, especially when the substituent represents a small portion of the molecule, for this situation the local electrophilicity can give a better resolution for the comparison between the sites of different species. In summary, either index is equally good for the analysis of individual molecules, while local softness and local electrophilicity are appropriate for comparing set of molecules. In a set of similar molecules where the substituent represents a small part, local electrophilicity is a better descriptor.

On the other hand, in terms of fragments, independently of the method used to define them, one can integrate the local electrophilicity over the region associated to a fragment in order to obtain the corresponding contribution to the electrophilicity index. Similarly we can use the condensed Fukui function $\left(f_{k}\right)$ in eq. 5 to obtain a condensed local electrophilicity for each fragment or atom within a molecule, $w_{k} \equiv \omega f_{k}$. In either case, the fragment or atomic contributions to the local electrophilicity add to the electrophilicity index, $\omega=\Sigma w_{k}$. This alternative use of the local electrophilicity can be very successful, like the similar cases of the Fukui function and local softness, where the condensed version is easier to interpret.

\section{Results}

In the literature the local electrophilicity index has been used to analyze the chemical reactivity of several sets of molecules [12]. In addition to the results reported in the literature, here we apply the local electrophilicity index to a family of substituted carbonyl compounds, some aldehydes and ketones. In this kind of molecules the carbon atom of the carbonyl group is electron deficient, an electrophilic site, and it is usually attacked by nucleophiles undergoing nucleophilic additions [13]. For the present study, the addition of water to the carbonyl group is analyzed. This addition is catalyzed either with acids or bases 
yielding a diol, $\mathrm{R}_{2} \mathrm{C}=\mathrm{O}+\mathrm{H}_{2} \mathrm{O} \longrightarrow \mathrm{R}_{2} \mathrm{C}(\mathrm{OH})_{2}$. The first step of the accepted mechanism corresponds to the rate determinant one. In this process, the nucleophile, a water molecule (acid catalyzed reaction) or a hydroxyl anion (base catalyzed), attacks the carbon atom of the carbonyl group. The second step corresponds to a proton exchange with the solvent to generate the final product. Therefore we can expect that the value of the local electrophilicity index at the carbon atom should be directly related to the propensity of the carbonyl group to react.

Formaldehyde, acetaldehyde, propanal, acetone, benzaldehyde and $\alpha$-chloroacetaldehyde were selected as a test set, trying to include different kind of substituents. All these molecules were optimized at B3LYP/6-311G** level. The corresponding negative and positive ions were calculated at the geometry of the neutral molecule with the same level of theory. Chemical potential and hardness are evaluated from the finite-difference scheme, $\mu=-(I+A) / 2$ and $\eta=I-A$, and they are used to obtain the values of the softness and the electrophilicity index. The condensed Fukui function, for an increase in the number of electrons, comes from the difference of the atomic charges, $f_{k}=$ $q_{k}^{(N+1)}-q_{k}^{(N)}$, where $q_{k}^{(N+1)}$ and $q_{k}^{(N)}$ correspond to the Mulliken atomic charge of the $k$-th atom of the anion and neutral molecule respectively. Mulliken populations were originally proposed to condense the Fukui function [14], however many other alternatives are available for the atomic charges. Even when every population analysis leads to different values, for the kind of molecules studied here, the trend is not modified because the carbonyl group is the polar part of the molecule. Most of the population schemes reproduce this feature. From the condensed Fukui function, the condensed form of the local indices follows, $s_{k}=S f_{k}$ and $w_{k}=\omega f_{k}$. Table 1 shows the values of some global quantities $\left(\mu, S, \Delta N_{\max }, \omega\right)$, the atomic charges on the atoms of the carbonyl group $\left(q_{\mathrm{C}}, q_{\mathrm{O}}\right)$ and the condensed local indices on the carbon atom $\left(f_{\mathrm{C}}, s_{\mathrm{C}}, w_{\mathrm{C}}\right)$ for the molecules of the test set. Equilibrium constants $\left(\log K_{\text {eq }}\right)$ for the addition reaction, taken from Ref. 13, are also included. These equilibrium constants correspond to the reaction in aqueous solution at $298 \mathrm{~K}$.

From Table 1 one can note that the carbon atom shows a positive charge in all the molecules of the set. All the other atoms have negative charges, except for the $\alpha$-chloroacetaldehyde, where the $\alpha$ carbon is also positive since it is bonded to a halogen. The donor site of the nucleophile corresponds to the oxygen atom, either for the water molecule or the hydroxyl an- ion, and it always has a negative atomic charge. Therefore, the reaction is initially dominated by the electrostatic interaction between the electrophilic and the nucleophilic sites. Differences must appear when charge transfer occurs.

For every molecule in the test set, the carbon atom shows the largest value of the condensed Fukui function and it can be characterized as the electrophilic center. In general, these values decrease with the size of the molecule, as one can note from Table 1, and this behavior comes from the normalization of the Fukui function. This effect is more evident in benzaldehyde, where the values of $f_{\mathrm{k}}$ are distributed both in the carbonyl and phenyl group and for this molecule the Fukui function of the carbon atom has the lowest value in the set. For this reason, it has been recognized that the Fukui function is not suitable for the comparison of molecules with different sizes [15]. Local softness also follows the same trend and this tendency mainly comes from the small range of variation of the molecular softness. In contrast, the condensed values of the local electrophilicity index have a different behavior. The comparison between the electronic reactivity parameters and the equilibrium constant for the addition reaction shows that, among this set of parameters, only the local electrophilicity index and the equilibrium constant follow the same trend, as expected. It is important to mention that the relationship is not linear. The local electrophilicity describes the propensity of a specific site to accept electrons. This information is relevant for the initial step of the reaction, but this index is unable to describe the electronic changes along the reaction path. Then, it differentiates the chemical behavior of a given sites in the set of molecules.

In our model system, the reaction involves two polar neutral molecules, where the negative site of water interacts with the positive site of the carbonyl group. It is important to note that the attraction between opposite charges is a long range interaction which is similar for the all the studied molecules. On the other hand, the tendency of the electrophilic site of carbonyl molecule to receive electrons from the other reactant differentiates the behavior of the carbonyl molecules.

\section{Concluding remarks}

Following a variational principle for the distribution of additional charge, we rationalize the use of the local extension of

Table 1. Reactivity parameters for some carbonyl compounds (electronic indices in atomic units).

\begin{tabular}{|c|c|c|c|c|c|c|c|c|c|c|}
\hline molecule & $-\mu$ & $S$ & $\Delta N_{\max }$ & $\omega$ & $q_{\mathrm{C}}$ & $q_{\mathrm{O}}$ & $f_{\mathrm{C}}$ & $\mathrm{s}_{\mathrm{C}}$ & $w_{C}$ & $\log K_{\mathrm{eq}}$ \\
\hline acetone & 0.140 & 2.354 & 0.330 & 0.0462 & 0.168 & -0.288 & 0.166 & 0.392 & 0.0077 & -2.86 \\
\hline benzaldehyde & 0.176 & 2.801 & 0.493 & 0.0867 & 0.225 & -0.287 & 0.137 & 0.385 & 0.0119 & -2.08 \\
\hline acetaldehyde & 0.149 & 2.246 & 0.334 & 0.0498 & 0.150 & -0.273 & 0.280 & 0.629 & 0.0140 & -0.08 \\
\hline$\alpha$-chloroacetaldehyde & 0.172 & 2.359 & 0.406 & 0.0699 & 0.177 & -0.260 & 0.249 & 0.586 & 0.0174 & 1.57 \\
\hline
\end{tabular}

$K_{\text {eq }}$ taken from Ref. 13 . 
the electrophilicity index. This quantity, which is proportional to the Fukui function, locally describes the distribution of the additional charge in an electronic system. The usefulness of the related local properties for different kinds of analysis is also discussed, as well as the corresponding condensed version. The local electrophilicity index is tested for an addition reaction, where the proposed index follows the trend of the reaction equilibrium constant for the set of molecules considered. This behavior confirms the physical meaning of this local quantity.

Although it is very tempting to use the Fukui function in the direction of decreasing number of electrons to extend the present work to define a nucleophilicity index, the framework is compromised with the process of accepting charge, and the nucleophiles do not behave in this way. Therefore we think that this problem should be approached from a different perspective [16]. An alternative is to use of a differentiated behavior for accepting and releasing electrons in the definition of electroaccepting and electrodonating powers [17].

Roy et al. have recently presented another definition of electrophilicity [18]. Therein, a relative electrophilicity index associated to site $k$ in the molecule was defined as the ratio $s_{k}{ }^{+} / s_{k}^{-}$, where $s_{k}{ }^{+}$and $s_{k}{ }^{-}$are regional (condensed to atom $k$ ) softnesses for the nucleophilic and electrophilic attacks, respectively. The representation of local electrophilicity to determine site selectivity using this index is less universal than the one presented here, eq. 5 , in the sense that the present approach applies to all atomic sites in the molecule. It is not necessarily restricted to those sites that present comparable values in the electrophilic and nucleophilic softness. A recent local reactivity index, the dual descriptor [19], is also related to Roy's [18], $\Delta f=f^{+}-f^{-}=\left(s^{+} / s^{-}-1\right) / f^{-}$, and its use is under exploration. Another advantage of the present local model is that it provides a normalized definition of the local electrophilicity.

\section{Acknowledgments}

We dedicate this work to Prof. José Luis Gázquez, a good friend and a good scientist. The support from FONDECYT contract No. 1030548, CONACYT project 155070, and CONACYT Cátedra Patrimonial for R. C. is kindly appreciated.

\section{References}

1. Parr, R. G.; Yang, W. Density Functional Theory of Atoms and Molecules. Oxford, New York, 1989.

2. See for example: Geerlings, P.; De Proft, F.; Langenaeker, W. Chem. Rev. 2003, 103, 1793- 1873. Gázquez, J. L. J. Mex. Chem. Soc. 2008, 52, 3-10. Chattaraj, P. K. (Ed.) Chemical Reactivity
Theory: A Density Functional View, Taylor \& Francis/CRC Press, Boca Raton, FL, 2009.

3. Parr, R. G.; Donnelly, R. A.; Levy, M.; Palke, W. E. J. Chem. Phys. 1978, 69,4431.

4. Parr, R. G.; Pearson, R. G. J. Am. Chem. Soc. 1983, 105, 7512.

5. Parr, R. G.; Yang, W. J. Am. Chem. Soc. 1984, 106, 4049.

6. Yang, W.; Parr, R. G. Proc. Natl. Acad. Sci. USA 1985, 82, 6723.

7. Berkowitz, M.; Parr, R. G. J. Chem. Phys. 1988, 88, 2554.

8. Maynard, A. T.; Huang, M.; Rice, W. G.; Covel, D. G. Proc. Natl. Acad. Sci. USA 1998, 95, 11578.

9. Parr, R. G.; von Szentpaly, L.; Liu, S. J. Am. Chem. Soc. 1999, 121, 1922.

10. a) Fuentealba, P.; Contreras, R., in: Reviews of Modern Quantum Chemistry. A Celebration of the Contributions of Robert G. Parr, Vol. 2, Sen, K. D., Ed., World Scientific, New Jersey, 2002, 10131052. b) Perez, P.; Toro-Labbé, A.; Aizman, A.; Contreras, R. $J$. Org. Chem. 2002, 67, 4747. c) Chattaraj, P. K.; Maiti, B.; Sarkar, U. J. Phys. Chem. A 2003, 107, 4973.

11. Ayers, P. W.; Parr, R. G. J. Am. Chem. Soc. 2000, 122, 2010.

12. See for example: Chamorro, E.; Chattaraj, P. K.; Fuentealba, P. J. Phys. Chem. A 2003, 107, 7068. Domingo, L. R.; Perez, P.; Contreras, R. J. Org. Chem. 2003, 68, 6060. Perez, P. J. Org. Chem. 2003, 68, 5886. Perez, P. J. Phys. Chem. A 2003, 107, 522. Padmanabhan, J.; Parthasarathi, R.; Sarkar, U.; Subramanian, V.; Chattaraj, P. K. Chem. Phys. Lett. 2004, 383, 122. Parthasarathi, R.; Padmanabhan, J.; Elango, M.; Subramanian, V.; Chattaraj, P. K. Chem. Phys. Lett. 2004, 394, 225. Parthasarathi, R.; Subramanian, V.; Roy, D. R.; Chattaraj, P. K. Bioorg. Med. Chem. 2004, 12, 5533. Perez, P. J. Org. Chem. 2004, 69, 5048. Roy, R. K. J. Phys. Chem. A 2004, 108, 4934. Chattaraj, P. K.; Roy, D. R. J. Phys. Chem. A 2005, 109, 3771. Elango, M.; Parthasarathi, R.; Narayanan, G. K.; Sabeelullah, A. M.; Sarkar, U.; Venkatasubramaniyan, N. S.; Supramanian, V.; Chattaraj, P. K. J. Chem. Sci. 2005, 117, 61. Meneses, L.; Fuentealba, P.; Contreras, R. Tetrahedron 2005, 61, 831. Roy, R. K.; Usha, V.; Paulovic, J.; Hirao, K. J. Phys. Chem. A 2005, 109, 4601. Roy, D. R.; Parthasarathi, R.; Padmanabhan, J.; Sarkar, U.; Subramanian, V.; Chattaraj, P. K. J. Phys. Chem. A 2006, 110, 1084. Chattaraj, P. K.; Sarkar, U.; Roy, D. R. Chem. Rev. 2006, 106, 2065.

13. Carroll, F. A. Reactions and Mechanisms in Organic Chemistry. Brooks/Cole, New York, 1998, Secc. 7.3.

14. Yang, W.; Mortier, W. J. Am. Chem. Soc. 1986, 108, 57085711.

15. See for example Ref. 14, Table II. In the series of methyl-substituted amines, the condensed Fukui function at the nitrogen atom decreases with the substitution, while the gas-phase basicity increases.

16. See for example: Cedillo, A.; Contreras, R.; Galván, M.; Aizman, A.; Andrés, J.; Safont, V. S. J. Phys. Chem. A 2007, 111, 24422447.

17. Gázquez, J. L.; Cedillo, A.; Vela, A. J. Phys. Chem. A 2007, 111, 1966-1970.

18. Roy, R. K.; Krishnamurti, S.; Geerlings, P.; Pal, S. J. Phys. Chem. A 1998, 102, 3746. Roy, R. K.; Pal, S.; Hirao, K. J. Chem. Phys. 1999, 110, 8236. Roy, R. K.; Tajima, N.; Hirao K. J. Phys. Chem. A 2001, 105, 2117.

19. Morell, C.; Grand, A.; Toro-Labbé, A. J. Phys. Chem. A, 2005, 109, 205. 